\title{
Angle and space multiplexed holographic storage using the $90^{\circ}$ geometry
}

\author{
Geoffrey W. Burr, Fai H. Mok, Demetri Psaltis \\ Department of Electrical Engineering, California Institute of Technology, Pasadena, CA 91125, USA
}

Received 26 July 1995

\begin{abstract}
The $90^{\circ}$ geometry - with reference and signal beams entering orthogonal crystal faces - is used to angularly multiplex up to 500 holograms at each of 8 spatially multiplexed locations in a $\mathrm{LiNbO}_{3}$ crystal. A segmented mirror array and a $2 \mathrm{D}$ mechanical scanner are used to perform both angular and spatial multiplexing. We show that this mirror array can be used with non-mechanical angle scanners, providing for holographic storage in multiple locations with no moving parts.
\end{abstract}

\section{Introduction}

Volume holography can be used to multiplex multiple pages of data by changing the angle [1], wavelength $[2,3]$, or phase code $[4,5]$ of the reference beam. The storage capacity $C$ achievable with each of these methods can be written as

$C=N M$,

where $N$ is the number of bits in each stored page, and $M$ is the number of superimposed pages. Assuming one bit per pixel, $N$ is limited to about $10^{6}$ by current spatial light modulator (SLM) technology. $M$ is limited to approximately $10^{4}$ by either the material dynamic range or the number of distinct reference angles (or wavelengths or phase codes) that can be generated. Total achievable storage capacity at one location is therefore between 1 and 10 gigabits. In order to increase the capacity beyond this limit, we can use spatial multiplexing in which angle, wavelength, or phase code multiplexed holograms are stored at multiple locations.
Spatial multiplexing has been used for holographic storage in both 2D and 3D media [6-12]. Two general methods have recently been proposed for accomplishing simultaneous spatial and angular multiplexing in 3D media. The first involves mechanically moving the storage medium to access different spatial locations $[10,11]$. The second method uses acoustooptic deflectors (AODs) to perform both angle and spatial multiplexing $[6-9,12]$, which leads to fast random access at the expense of a more complex optical system.

The first to demonstrate non-mechanical spatial multiplexing was Huignard et al. in the early 1970's $[7,8]$. Their system used two cascaded AOD deflectors to supply the same 2D spatial translation to both the reference and object arms. A reflection grating combined the beams at the crystal surface. Angle multiplexing at each location was performed by a mechanical rotating beam shearing element placed in the reference arm. Using this arrangement, they were able to store 10 holograms at each of 25 locations.

The key element in our realization of angle and spatial multiplexing is a segmented mirror array, which 
preserves horizontal angular multiplexing while using vertical deflection to step between distinct storage locations. In this paper, we report on the use of such a mirror array to store 500 angle multiplexed holograms at each of 8 spatially multiplexed locations using a novel $90^{\circ}$ storage geometry. First, we describe the experimental setup, including the mechanical scanner and the operation of the mirror array. We then list the experimental parameters and show reconstructions of stored holograms. The restrictions present in this system are explored, including the absorption in the long crystal bar. We show that although this absorption causes the modulation depth in the $90^{\circ}$ geometry to be position dependent, the effect is canceled out upon readout for low modulation depth. Finally, we discuss using spatial multiplexing over a 2D grid for increased storage capacity, implementing non-mechanical scanners for high speed random access to the stored holograms, and readout rate-diffraction efficiency considerations.

\section{System description}

The experimental setup is shown in Fig. 1. The recording medium is a $0.01 \% \mathrm{Fe}$-doped $\mathrm{LiNbO}_{3}$ bar of dimensions $8 \mathrm{~mm} \times 8 \mathrm{~mm} \times 50 \mathrm{~mm}$. The $c$-axis is in the horizontal plane and at $45^{\circ}$ with respect to the vertical faces of the bar. Holograms are recorded in the $90^{\circ}$ geometry with the signal beam propagating down the long axis of the bar and the reference entering from the side. This configuration is convenient because reference and signal enter normal to a crystal face. Also, since the object beam traveling along the long dimension of the crystal is present at all spatial locations, there is no need to deflect the object beam.

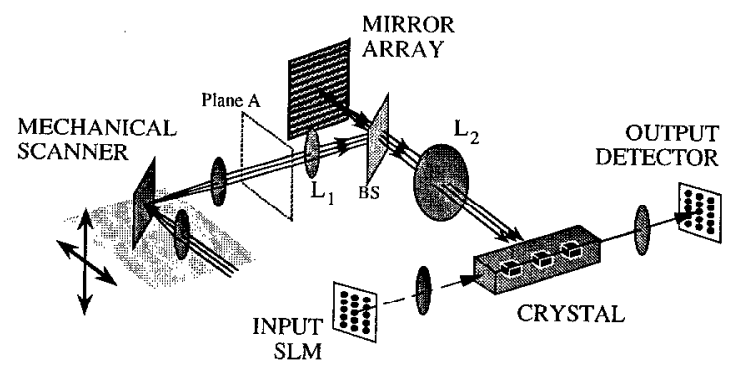

Fig. 1. Experimental setup for demonstration of spatial and angular multiplexing with the $90^{\circ}$ geometry.
Input images are presented on a fixed input SLM and reconstructions observed on a fixed $2 \mathrm{D} C \mathrm{CD}$ array at the other end of the bar, no matter which location is being accessed. In the experiment, the SLM was a transparency mounted on a rotational stage. The reference arm consists of a mechanical scanner, a segmented mirror array, and several lenses. The scanner focuses the input beam to a spot with a single lens and reflects the light off a mirror oriented at $45^{\circ}$ with respect to the optical axis of the lens. The position of the focussed spot can be scanned in both dimensions by moving the lens and mirror assembly with two computer controlled actuators. Note that horizontal translation is achieved by moving the assembly in the axial direction, so there is no limit on movement in this dimension. A periscope arrangement can be used to achieve the same effect in both dimensions, but this was not necessary in this experiment. A pair of lenses images the focussed spot onto the mirror array.

The mirror array is composed of multiple horizontal strips, stacked vertically. The operation of the mirror strips is shown in Figs. 2a and 2b. Each mirror strip has a unique orientation. When the focussed spot reflects off a particular mirror strip, it is deflected horizontally by twice the mirror tilt angle. The reflected beam is then directed towards the crystal through the

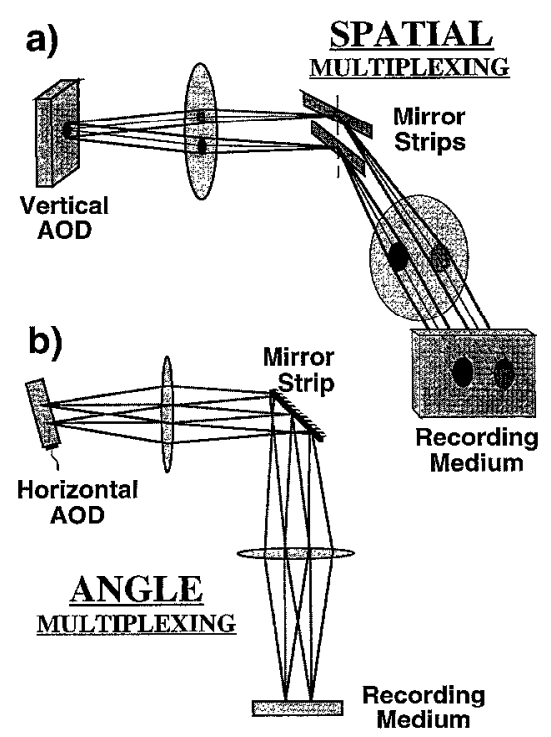

Fig. 2. Operation of mirror array segments. (a) Selection of spatial location by vertical beam deflection. (b) Selection of incident reference beam angle by horizontal beam deflection. 


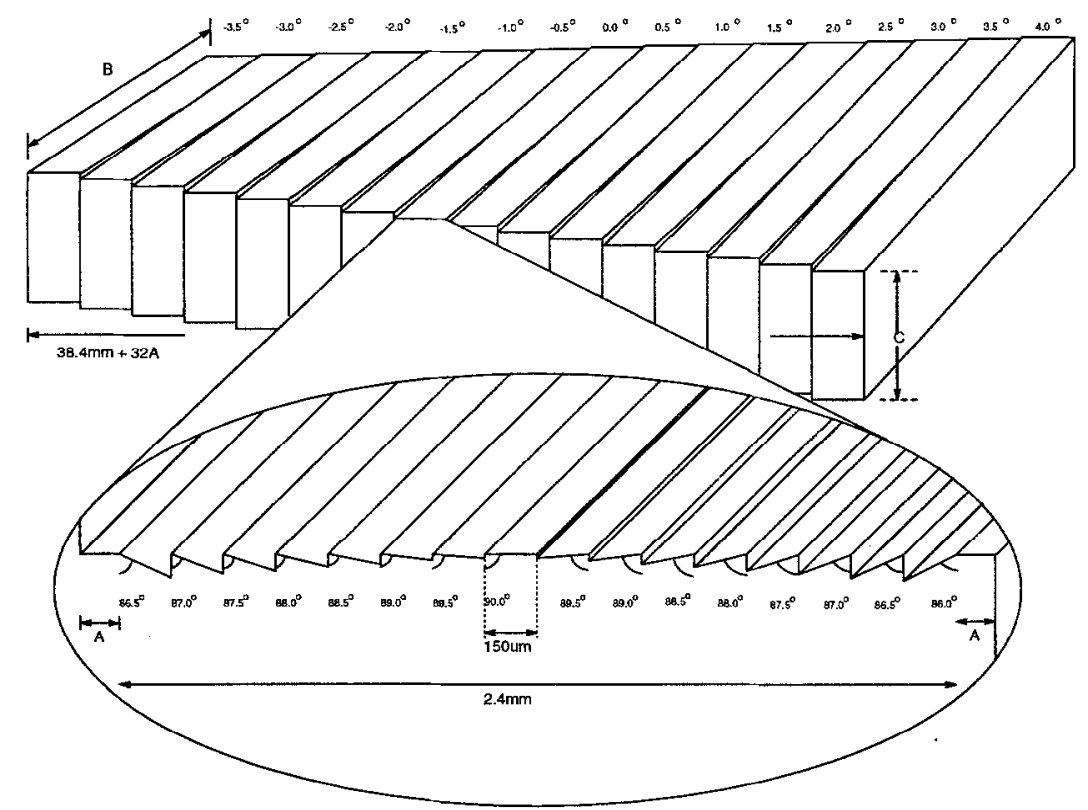

Fig. 3. Schematic of segmented mirror array, showing horizontal and vertical tilt angles.

beamsplitler (not shown in Fig. 2). A Fourier transform lens converts the focussed spot into a collimated beam at the crystal. The spatial location of the collimated beam is determined by the deflection angle of the mirror element (Fig. 2a), while the horizontal angle of incidence is determined by the horizontal position of the focussed spot on the mirror strip (Fig. 2b). In this way, the 2D mechanical scanner selects the position and incidence angle of the reference beam at the crystal surface. The beamsplitter is required because the surface of the mirror array must be aligned with the focal plane (the center of the 4-F system containing lenses $L_{1}$ and $L_{2}$ ), in which the converging beams achieve their focus. This allows us to minimize the vertical size of each mirror strip, yet still avoid crosstalk to other storage locations. Note also that this mirror array can be used to combine spatial multiplexing with either wavelength or phase-code multiplexing.

The mirror array used in the experiment was designed for spatial multiplexing over a 2D grid [12]. A schematic of the mirror array is shown in Fig. 3. It is composed of 16 "tiles", each of which has 16 mirror strips cut as grooves. The change in angle between tiles and between groove faces is $0.5^{\circ}$. The angular change between tiles is orthogonal to the inclination of the grooves, allowing tilting of individual facets in both directions. The mirror array was fabricated using standard blazed grating technology. This technique involves cutting grooves with a diamond tip in a gold surface on a brass substrate. The groove angles are controlled by the tilt of the tip with respect to the substrate, while the groove width is set by the dimensions of the tip. Each strip is $150 \mu \mathrm{m}$ wide and $75 \mathrm{~mm}$ long. A photograph of the fabricated array is shown in Fig. 4.

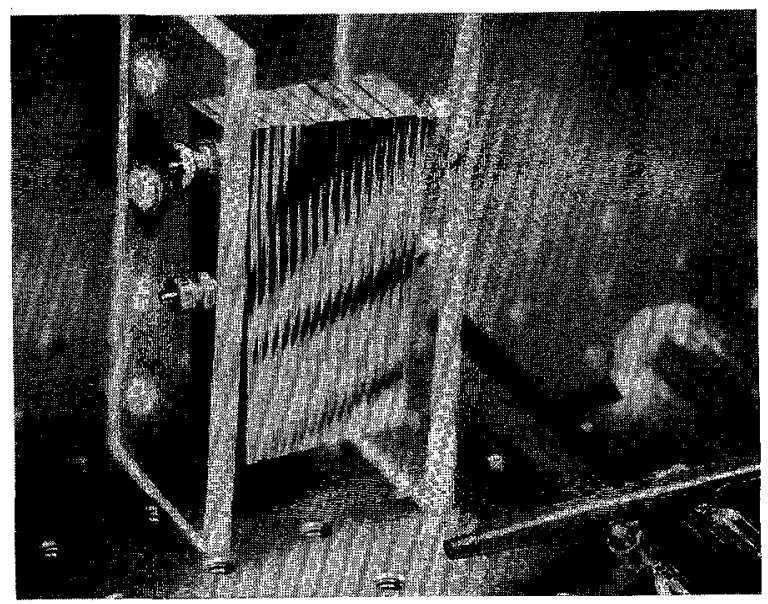

Fig. 4. Photograph of segmented mirror array, manufactured with blazed grating technology. 

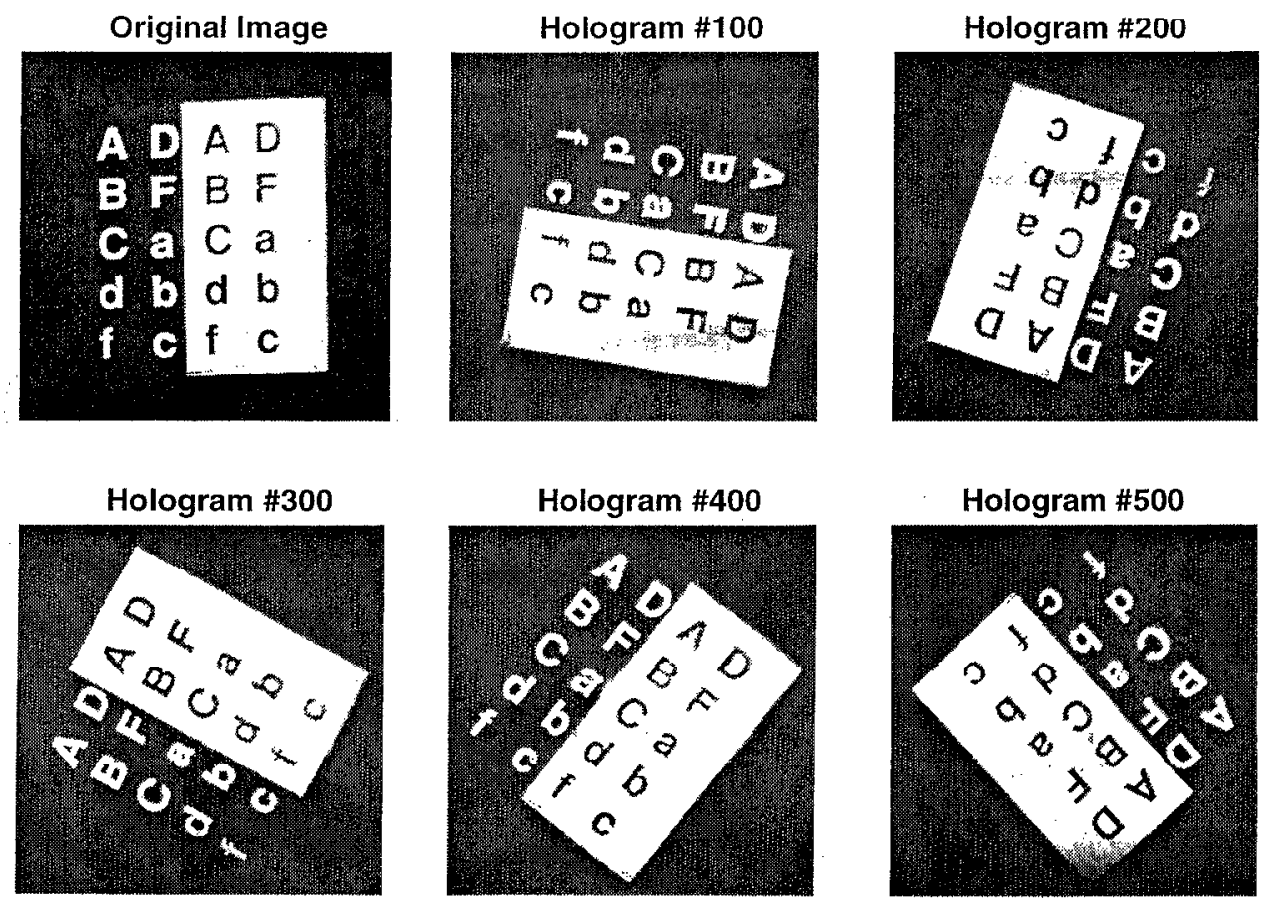

Fig. 5. Example reconstructions from storage of 500 holograms at one spot.

\section{Experiment}

First, 500 holograms were stored at a single location with a $6 \mathrm{~mm}$ diameter reference beam. An exposurc schedule [13] was used to equalize the diffraction efficiencies of the holograms. The longest exposure was $12.2 \mathrm{~s}$ and the shortest $1.65 \mathrm{~s}$. The total recording intensity incident on the crystal was $130 \mathrm{~mW} / \mathrm{cm}^{2}$, the ratio of the reference to signal beam intensity incident on the crystal was 6.25 , and the average diffraction efficiency $10^{-8}$. Several reconstructions are shown in Fig. 5. The original transparency is shown at upper left, and was rotated $1^{\circ}$ between each exposure.

Next, holograms were recorded at each of 8 spatially multiplexed spots (each reference beam was 4 $\mathrm{mm}$ in diameter) along the length of the crystal, using 8 of the 256 mirror strips on the mirror array. At locations near the center of the crystal, 500 holograms were stored at each location. Towards the ends of the bar, the limited aperture of the beamsplitter restricted us to 350 holograms per location. The average diffraction efficiency was again $10^{-8}$. Several reconstructions are shown in Fig. 6 . Note that, in the reconstructions of the holograms stored towards the ends of the bar, the top and bottom edges are missing. This occurred because the reference beam becomes oval shaped at these outermost storage locations, due to lens aberrations and the inclination of the mirror strip out of the focal plane as the focussed spot moves off-axis.

In our experiment, the limit on the number of holograms per location was the number of distinct reference angles we could provide to each location, rather than crystal dynamic range. This limit was imposed by the beamsplitter aperture, not the mirror array. We only used $28 \mathrm{~mm}$ (about $37 \%$ ) of the horizontal extent of the mirror array. Therefore, an optical system with larger apertures can access 1000 holograms per spot, or more. Ultimately, for a given laser power, the dynamic range of the system limits the number of holograms per location, since the diffraction efficiency is inversely proportional to the number of holograms squared [13]. In this system there are additional losses not normally encountered in a standard angle multiplexing holographic setup, bringing signal levels closer to the fixed detector noise floor and reducing dynamic range. These losses include the optical loss 

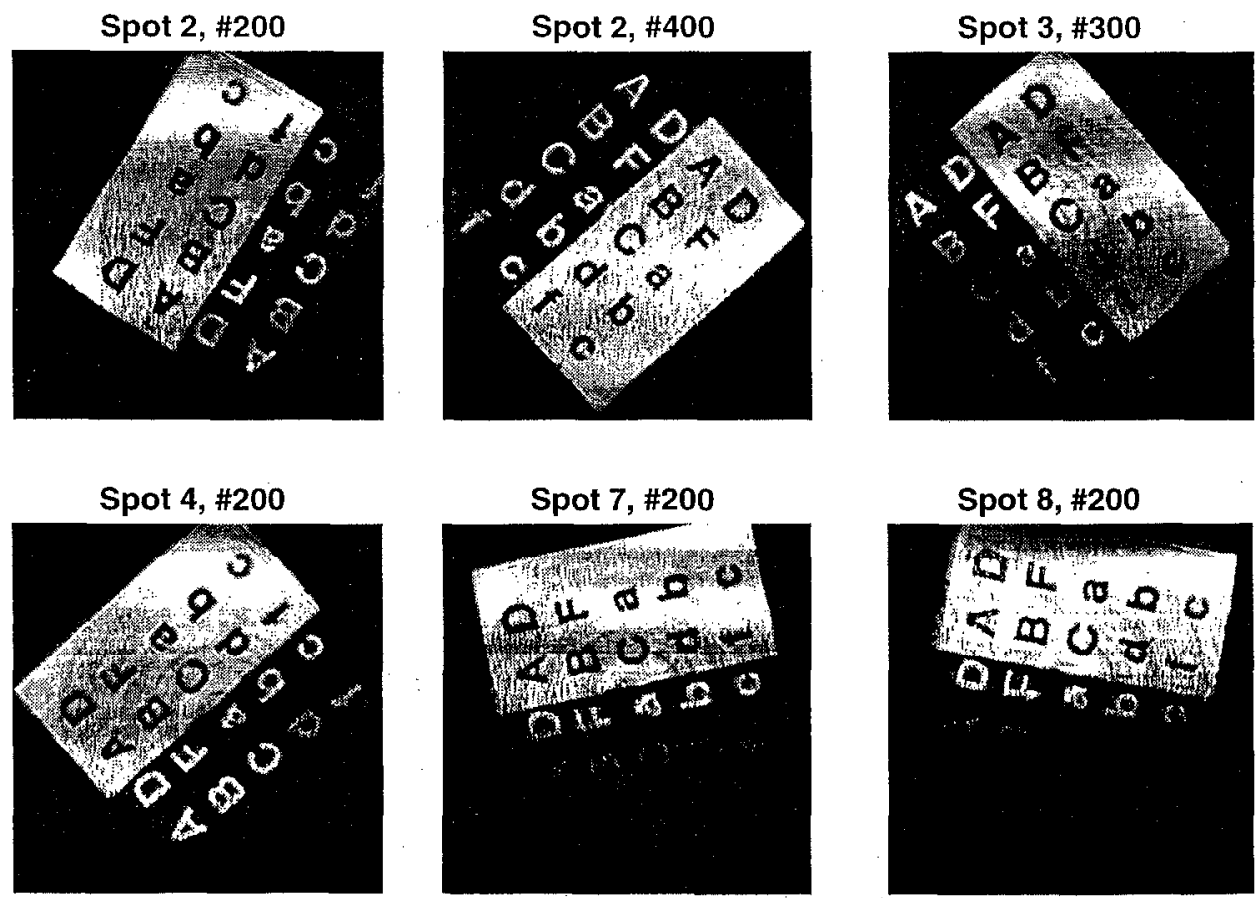

Fig. 6. Example reconstructions from storage of up to 500 holograms at each of 8 spots.

at the nonpolarizing beamsplitter, the low reflectivity $(40 \%)$ of the gold surface of the mirror array, and absorption in the long crystal. Absorption allows approximately $5 \%$ of the light illuminating one of the end faces to be transmitted out the other end.

\section{Position-dependent modulation depth}

Another effect of the absorption in the long bar is that the modulation depth during storage will vary from location to location as well as across each hologram location. We will show that this position dependent modulation depth does not lead to a non-uniform

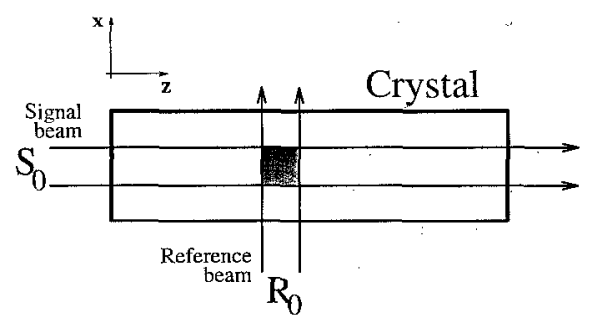

Fig. 7. The $90^{\circ}$ geometry. output image because absorption is also present upon readout. When we interfere two plane waves in the $90^{\circ}$ geometry, the resulting grating strength at any location $(x, y, z)$ (see Fig. 7) is proportional to the modulation depth. With a strong reference beam $R_{0} \exp (-\alpha x)$ and a weak signal beam $S_{0} \exp (-\alpha z)$, we can write the perturbation in the dielectric constant as

$$
\Delta \epsilon(x, y, z) \propto \frac{2 R_{0} S_{0} \mathrm{e}^{-\alpha(x+z)}}{R_{0}^{2} \mathrm{e}^{-2 \alpha x}+S_{0}^{2} \mathrm{e}^{-2 \alpha z}} \sim \frac{2 S_{0} \mathrm{e}^{\alpha(x-z)}}{R_{0}},
$$

where $2 \alpha$ is the absorption coefficient. When reconstructing the hologram, the readout bean must first propagate to position $(x, y, z)$, suffering an attenuation of $\exp (-\alpha x)$. The amplitude contributed to the scattered wave by the grating at this point is proportional to the grating strength $\Delta \epsilon(x, y, z)$. Then, neglecting rediffraction, we need only consider the additional loss in moving from $(x, y, z)$ to the far end of the crystal. For Bragg-matched readout, the scattered waves from all volume elements are in phase and we have 


$$
\begin{aligned}
& \sqrt{P_{\text {out }}} \propto 2 S_{0} \int_{V} \mathrm{e}^{\alpha(x-z)} \mathrm{e}^{-\alpha x} \mathrm{e}^{-\alpha(L-z)} \mathrm{d} r \\
& \sim 2 S_{0} V \mathrm{e}^{-\alpha L},
\end{aligned}
$$

where $L$ is the length of the crystal and $V$ the volume occupied by the grating.

Another consequence due to absorption is the dependence of the time constant upon total intensity, which decays in the $x$ direction if we assume that most of the intensity is contributed by the reference beam. Therefore, the far side of the grating - the portion on the opposite side from the entrance of the reference beam - will be written more slowly than the near side. However, it will be erased at a commensurately slower rate. Mathematically, we can represent the equalized diffraction efficiency after scheduled recording [13] as

$$
\eta \sim\left(\frac{A_{0}}{\tau_{\mathrm{r}}} \tau_{\mathrm{e}}\right)^{2} \frac{1}{M^{2}},
$$

where $A_{0}$ is the saturation grating amplitude, $\tau_{\mathrm{r}}$ and $\tau_{\mathrm{e}}$ are the recording and erasure time constants, and $M$ is again the number of holograms. If $\tau_{\mathrm{e}}$ and $\tau_{\mathrm{r}}$ have the same dependence upon total intensity, the effect of any position dependent time constant will be cancelled out by the operation of scheduled recording.

One final aspect to be considered is the presence of the object beam at all locations, including those where holograms have already been written. Holograms at the location which is first used for storage will be erased by the object beam during exposure of all subsequent holograms. This does not cause much difficulty in the system described above for two reasons. One reason is that the external ratio of reference to signal intensity is large, approximately 6.25 . In addition, the storage locations can be filled starting from the end farthest from the entry of the object beam. In this way, the holograms exposed to the object beam for the longest time are buffered from its effects by the absorption of the long crystal. For these two reasons, very little erasure of the holograms occurs once the reference beam moves to the next storage location.

\section{Discussion}

The fact that the amplitude of the diffracted light is independent of the location of the grating volume, $V$, implies that each volume element of a stored hologram contributes equally to the resulting effective diffracted power and images are reconstructed uniformly. We also conclude that the output power is independent of the position of the hologram within the crystal. If we extend the length of the crystal, we can increase the number of spatial locations. If, simultaneously, we decrease $\alpha$ to maintain the same absorption loss, the diffraction efficiency remains the same. The limit to the storage capacity of this architecture is then the aperture of the lens providing the reference beam from the mirror array. For example, if we use a lens with a $130 \mathrm{~mm}$ aperture and a $200 \mathrm{~mm}$ focal length, we could accommodate approximately 30 locations of $3 \mathrm{~mm}$ diameter in a $100 \mathrm{~mm}$ long bar. The trade-off for the incrcased storage capacity is a decrease in the recording speed, since a less absorptive crystal is slower.

Another way to increase capacity is to perform spatial multiplexing in 2D $[9,12]$. The existing mirror array is already fabricated to provide spatial multiplexing over a 2D grid since the mirror strips have both vertical and horizontal tilts. We can extend the capacity of the system presented above by simply stacking bars vertically. The only additional complication is the required vertical scanning of the object beam to the appropriate storage row. This could be implemented by illuminating the input SLM in the object arm with a plane wave at the appropriate incidence angle. The Fourier transform of the input page will then be presented for storage at the bar corresponding to the vertical illumination angle. For example, if 16 bars are vertically stacked with 1000 holograms stored at each of 16 locations on each bar (the $16 \times 16$ limit is due to the current mirror array), then the capacity is 256000 holograms.

In the experiment described above, we used a mechanical scanner to move a focussed spot and a pair of lenses to image this moving spot onto the surface of the mirror array. In the center of this pair of lenses (plane A in Fig. 1), there exists a plane wave centered on the optical axis with a vertical and horizontal incidence angle. We can create an identical plane wave by using a $2 \mathrm{D}$ non-mechanical angle scanner in this plane A. One such example is two crossed AOD cells with cylindrical accessing lenses [7,8]. The Doppler shift added by these cells can be removed by an electrooptic modulator. In this way, the mirror array can provide spatially multiplexed holographic storage without 
mechanical movement.

Using mechanical scanners, the access time to any hologram depends on the movement required plus an additional settling time. With a 2D non-mechanical angle scanner and the mirror array, we can present the correct reference beams to the crystal independently of the order in which holograms are accessed. The random access time to any one of the stored holograms is then limited by detector sensitivity. For instance, if the read-out beam is $1 \mathrm{~W} / \mathrm{cm}^{2}$ at the crystal surface and we need 1000 photogenerated electrons per detector pixel, the detector-limited random access time in the current system $\left(\eta \sim 10^{-8}\right)$ is approximately $40 \mathrm{~ms}$. If we require an access time of $100 \mu \mathrm{s}$, then the diffraction efficiency necessary becomes $4 \times 10^{-6}$. This sort of increase in holographic diffraction efficiency can be achieved through appropriate annealing of the $\mathrm{LiNbO}_{3}$ crystal [14]. With this improvement, the system will become limited by the detector readout rate.

\section{Acknowledgements}

This research is supported by RADC/IRAP. G. Burr acknowledges support of a U.S. Office of Naval Research graduate fellowship.

\section{References}

[1] D.L. Staebler, J.J. Amodei and W. Phillips, VII Intern. Quantum Electron. Conf., Montreal, May (1972).

[2] F.T.S. Yu, S. Wu, A.W. Mayers and S. Rajan, Optics Comm. 81 (1991) 343.

[3] G.A. Rakuljic, V. Leyva and A. Yariv, Optics Lett. 17 (1992) 1471.

[4] T.F. Krile, M.O. Hagler, W.D. Redus and J.F. Walkup, Appl. Optics 18 (1979) 52.

[5] J.E. Ford, Y. Fainman and S.H. Lee, Optics Lett. 15 (1990) 1088.

[6] L. d'Auria, J.P. Huignard and E. Spitz, IEEE Trans. Mag. MAG-9 (1973) 83.

[7] L. d'Auria, J.P. Huignard, C. Slezak and E. Spitz, Appl. Optics 13 (1974) 808.

[8] J.P. Huignard, F. Micheron and E. Spitz, Optical Properties of Solids, ch. 16, (North Holland, Amsterdam, 1976) p. 847.

[9] F. Mok, D. Psaltis and G.W. Burr, SPIE - San Diego (1992), Proc. SPIE 1773c, p. 1.

[10] D. Psaltis, Byte, Sep 1992, p. 179.

[11] S. Tao, D.R. Selvah and J.E. Midwinter, Optics Lett. 18 (1993) 912

[12] G.W. Burr, F. Mok and D. Psaltis, SPIE - San Diego (1993), Proc. SPIE 2026-62.

[13] D. Psaltis, D. Brady and K. Wagner, Appl. Optics 27 (1988) 1752.

[14] F.H. Mok, Optics Lett. 18 (1993) 915. 